\title{
Der Kardiologe
}

\section{Wie lautet Ihre Diagnose?}

Kardiologe 2021 · 15:282-285

https://doi.org/10.1007/s12181-021-00459-8

Angenommen: 28. Januar 2021

Online publiziert: 18. Februar 2021

(c) Springer Medizin Verlag $\mathrm{GmbH}$, ein Teil von Springer Nature 2021

\section{Vorgeschichte}

Ein 45-jähriger Mann mit kurzer, jedoch komplexer kardiologischer Vorgeschichte stellte sich wegen zunehmender Dyspnoe erneut stationär in unserer Klinik vor. Erstmalig wurde der Patient 4 Monate zuvor wegen einer Synkope bei uns untersucht. Die transthorakale Echokardiographie (TTE) hatte damals ein mittelgradiges, kombiniertes Aortenklappenvitium ergeben mit normaler linksventrikulärer Ejektionsfraktion (LVEF). Mittels Koronar-CT wurde eine relevante koronare Herzerkrankung ausgeschlossen. Ein Hinweis auf eine kardiogene Synkope ergab sich insgesamt nicht. Drei Wochen später wurde der Patient mit Fieber und einem ST-Hebungsinfarkt erneut vorstellig. Zunächst bestand der Verdacht auf eine COVID-19-Infektion, es wurde jedoch umgehend eine Koronarangiographie durchgeführt. Der Ramus circumflexus (RCX) war peripher thrombotisch verschlossen bei Linksversorgungs-

Vanessa Rubesch-Kütemeyer · Arianit Pula · Marios Vlachojannis · Stephan Gielen

Klinik für Kardiologie, Angiologie und internistische Intensivmedizin, Klinikum Lippe Detmold, Detmold, Deutschland

\section{Der lange Weg zu einer selten gewordenen Komplikation des akuten Myokardinfarktes}

typ und ansonsten unauffälligen Koronararterien. Es erfolgte eine PTCA mit 3,0/29 mm Drug-Eluting-Stent-Implantation. Die Door-to-Balloon-Zeit betrug 79 min. Die maximale Kreatinkinasekonzentration lag nach $24 \mathrm{~h}$ bei $2561 \mathrm{U} / \mathrm{l}$ und war stetig rückläufig. Bei persistierendem Fieber, steigenden Infektwerten und negativem COVID-19-Abstrich wurde eine erweiterte Fokussuche durchgeführt. Es stellte sich in der TTE eine jetzt hochgradige Aortenklappeninsuffizienz dar. Die LVEF war bei Hypokinesie inferior und inferoseptal mit 45-50\% nun leicht eingeschränkt. Bei Nachweis von Escherichia coli und Enterococcus faecalis in der Blutkultur erfolgte anschließend eine transösophageale Echokardiographie, in der sich die Verdachtsdiagnose Aortenklappenendokarditis mit beginnendem Aortenwurzelabszess bestätigte. Bei unauffälligem Koronar-CT wenige Wochen zuvor handelte es sich initial also mit hoher Wahrscheinlichkeit um eine septische Embolie in den RCX. Der Patient wurde notfallmäßig zur Operation verlegt. Er erhielt einen mechanischen Aortenklappenersatz $(21 \mathrm{~mm})$ mit Patchplastik der Aortenwurzel. Postoperativ erfolgte eine zeitnahe Rückverlegung in unser Haus und nach Abschluss der antibiotischen Therapie die Verlegung zur Anschlussheilbehandlung.

\section{Aktueller Aufnahmebefund}

Bei der aktuellen Wiedervorstellung insgesamt 10 Wochen nach der Operation gab der Patient seit einiger Zeit eine zunehmende Belastungsdyspnoe und Orthopnoe, jetzt NYHA IV, und thorakale Schmerzen an. Insgesamt habe er sich nach der Anschlussheilbehandlung nicht wesentlich gestärkt gefühlt. Der Patient befand sich klinisch in einem deutlich reduzierten Allgemeinzustand. Der Auskultationsbefund war unauffällig. Die Vitalparameter waren ebenso unauffällig mit einem Blutdruck von
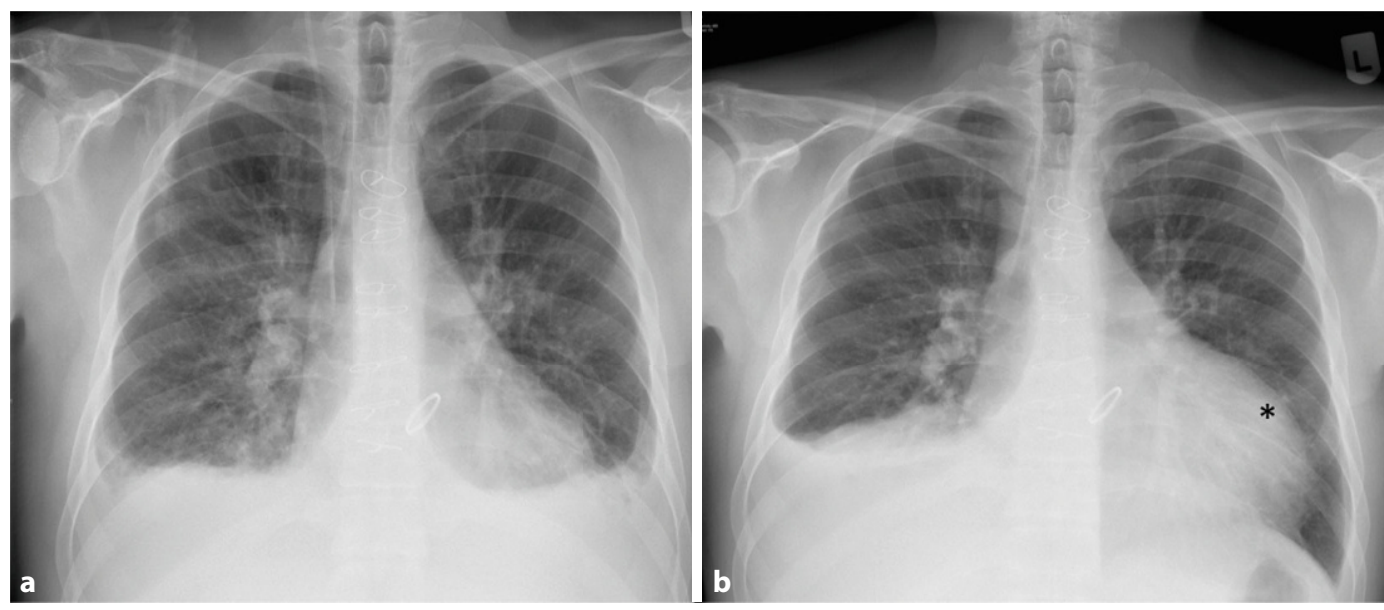

Abb. $1 \varangle$ a Röntgen postoperativ nach Aortenklappenersatz und $\mathbf{b}$ bei der letzten Vorstellung. Neu ist die Dilatation des LV (Asterisk), der Pleuraerguss rechts bleibt bestehen, links hat sich dieser in b zurückgebildet. Des Weiteren ist der Aortenklappenersatz in beiden Bildern zu erkennen 


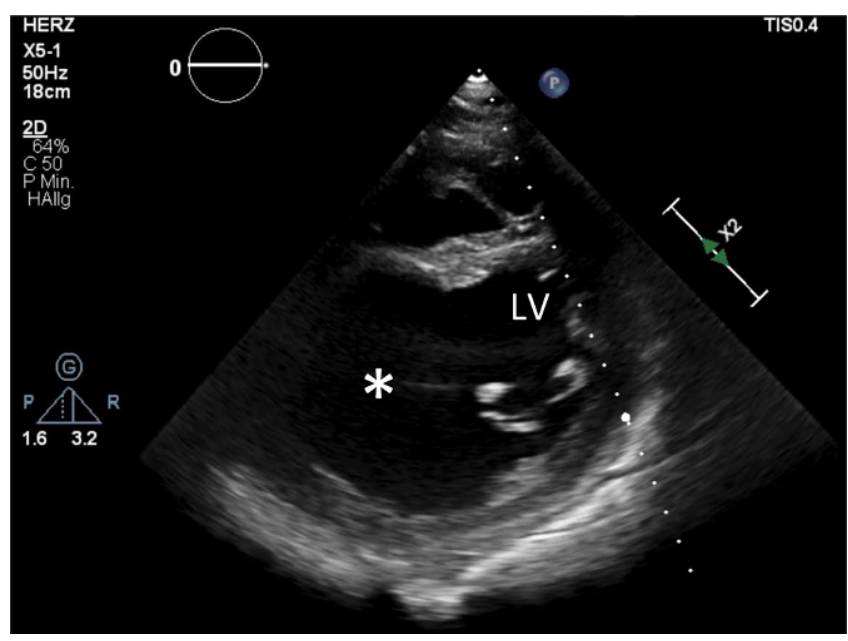

Abb. 2 ム TTE, parasternal kurze Achse des linken Ventrikels ( $L V$ ) auf Höhe der Mitralklappe. Es zeigt sich ein großer Defekt mit echoarmer Höhle inferoseptal und inferior (Asterisk)

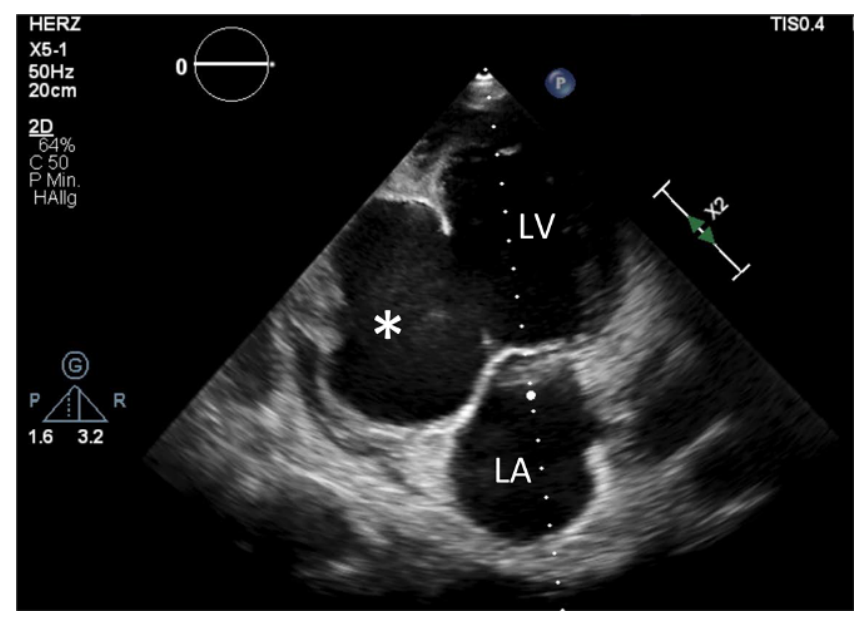

Abb. $3 \Delta$ TTE, 2-Kammer-Blick. Darstellung von linkem Ventrikel ( $L V$ ) und linkem Vorhof $(L A)$. Das Aneurysma (Asterisk) wurde maximal mit $78 \times 67 \mathrm{~mm}$ vermessen, der Defekt der basal- bis mitinferioren Wand misst $31 \mathrm{~mm}$
120/90 mm Hg, Herzfrequenz 99/min, Temperatur $36,2^{\circ} \mathrm{C}$.

Untersuchungen. Pathologische Werte: Natrium $131 \mathrm{mmol} / \mathrm{l}$, GOT $153 \mathrm{U} / \mathrm{l}$, GPT $134 \mathrm{U} / \mathrm{l}, \mathrm{LDH} 423 \mathrm{U} / \mathrm{l}$, CRP $1,6 \mathrm{mg} / \mathrm{dl}$, Hs-Troponin $57 \mathrm{pg} / \mathrm{ml}$. Procalcitonin normwertig. Im EKG fand sich eine Sinustachykardie bei Steiltyp, T-Negativierungen in II, III, aVF und V4-6 bei normalem R-Progress mit Zeichen der LV-Hypertrophie ohne ST-Streckenveränderungen. Die Thoraxröntgenaufnahme zeigte einen zunehmenden, rechts- seitigen Pleuraerguss, keine pulmonale Stauung, aber im Vergleich zur postoperativen Voraufnahme eine zunehmende Herzverbreiterung ( $\bullet$ Abb. 1). Eine TTE ergab folgende Befunde (• Abb. 2 und 3 ).

\section{Wie lautet Ihre Diagnose?}

\section{Weitere Untersuchungen}

Die TTE ergab zusätzlich eine jetzt hochgradig reduzierte LVEF des verbliebenen Ventrikels um $30 \%$ und eine begleitende mittelgradige Mitralklappeninsuffizienz. Die Aortenklappenprothese war unauffällig. Die kardiale CT-Untersuchung bestätigte den Befund eines Hinterwandaneurysmas von $78 \times 62 \mathrm{~mm}$ im Kurzachsenschnitt und $86 \mathrm{~mm}$ in der langen Achse (- Abb. 4 und 5).

\section{》) Diagnose: Großes \\ Ventrikelaneurysma der linksventrikulären Hinterwand}

Koronarangiographisch fand sich der dazu passende, ältere Verschluss des RCX im Stent. Ursache und Zeitpunkt des Stentverschlusses bleiben letztlich unklar. Der Patient war mit Phenprocoumon und Clopidogrel nach der Operation zurückübernommen worden.
Aufgrund der akuten Symptomatik mit kardialer Dekompensation, Ruhedyspnoe, sowie der Größe des Aneurysmas stellten wir den Patienten umgehend zur chirurgischen Aneurysmaresektion vor.

\section{Definition und Hintergrund}

Die Ausbildung eines Ventrikelaneurysmas ist in Zeiten der frühen Revaskularisierung mittels PTCA eine seltene Komplikation des akuten Myokardinfarktes geworden $[1,4]$.Zu unterscheiden sind 2 Entitäten. Die häufigere Variante ist das echte Aneurysma, das durch einen transmuralen Myokardinfarkt und konsekutive Fibrosierung und Ausdünnung der gesamten Wanddicke auf $<5 \mathrm{~mm}$ entsteht und häufig mit einem muralen Thrombus sowie im Verlauf entstehenden Verkalkungen vergesellschaftet ist. Prädilektionsstelle ist die Vorderwand und das vorderwandnahe Septum beim klassischen Verschluss des Ramus in- terventricularis anterior [2, 4]. Echte Aneurysmen entwickeln sich in der Regel innerhalb der ersten 8 Wochen nach Myokardinfarkt [4]. Die zweite seltenere, aber auch gefährlichere Entität ist das Pseudoaneurysma. Dieses entsteht durch eine Ruptur des LV, allerdings wird die Blutung durch das Perikard oder Verwachsungen gedeckt gehalten. Die Unterscheidung zwischen diesen beiden Formen ist wichtig, da die Rupturgefahr von Pseudoaneurysmen deutlich höher eingeschätzt wird, aber die Differenzierung klinisch trotz Bildgebung nicht immer möglich ist [1-3]. Inferiore Infarkte resultieren doppelt so häufig in Pseudoaneurysmabildung verglichen mit anterioren Infarkten. Pseudoaneurysmen sind entsprechend häufiger posterior, lateral, apikal oder inferior zu finden [2]. Das Symptomspektrum reicht von asymptomatisch bis zu einer schweren Herzinsuffizienz, thrombembolischen Komplikationen oder ventrikulären Tachykardien. Als 


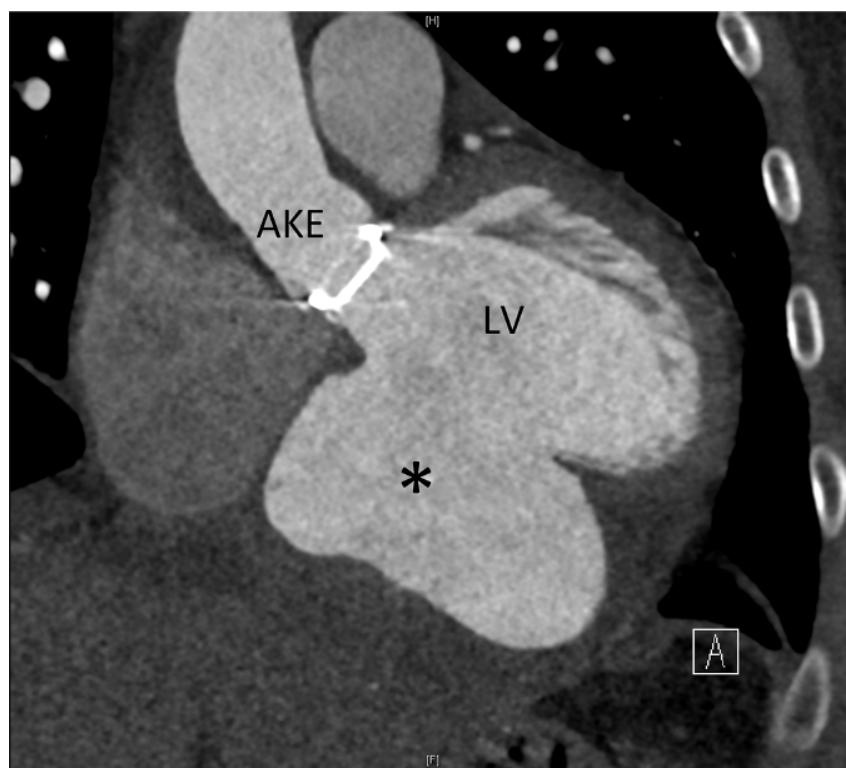

Abb. $4 \Delta$ Kardio-CT, 3-Kammer-Blick. Abgebildet sind hier unter anderem das Aneurysma (Asterisk), linker Ventrikel (LV) und gut erkennbar der mechanische Aortenklappenersatz ( $A K E$ )

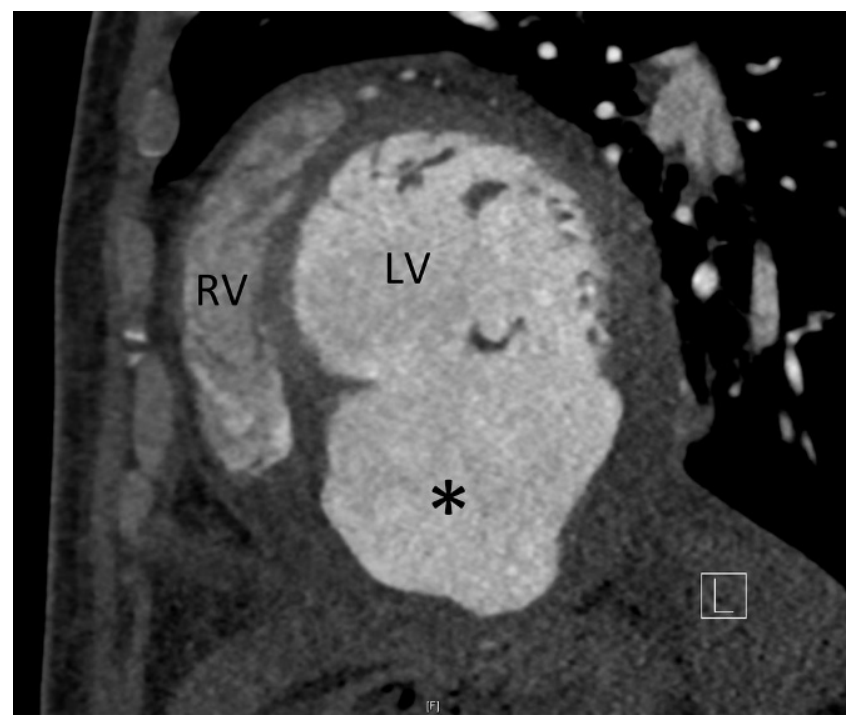

Abb. 5 - Kardio-CT kurze Achse des LV. Abgebildet sind hier unter anderem das Aneurysma (Asterisk), linker Ventrikel (LV) und ein Anschnitt des rechten Ventrikels bzw. des rechtsventrikulären Ausflusstraktes ( $R V)$
Maximalvariante besteht insbesondere bei Pseudoaneurysmen die Gefahr des plötzlichen Herztodes durch Ruptur [4]. Grundlegende Hinweise, dass es zu einer Aneurysmabildung gekommen ist, können typische EKG-Veränderungen wie nach Myokardinfarkt persistierende STHebungen über der Vorderwand geben, jedoch gibt es keine sicher spezifischen Symptome oder Zeichen zur Erkennung eines Aneurysmas oder Pseudoaneurysmas [1-3]. EKG und Thoraxröntgenaufnahme sind aber in einer Mehrzahl der Fälle zumindest auffällig [2]. Die TTE ist aufgrund der breiten Verfügbarkeit in der Regel die erste diagnostische Methode, die primär zum Einsatz kommt. Danach sollten kardiale CT- oder MRTUntersuchungen und ggf. eine LVAngiographie erfolgen. Die Therapie kann von konservativ medikamentös bei apikalen Vorderwandaneurysmen bis zum chirurgischen oder interventionellen Verschluss reichen, abhängig unter anderem von Aneurysmagröße, Entwicklung der LVEF und Ausprägung der Herzinsuffizienzsymptomatik [1-4]. Pseudoaneurysmen sollten in der Regel wegen der Gefahr einer Ruptur zeitnah operativ saniert werden, unbehandelt haben diese eine Mortalität bis $50 \%$ im Gegensatz zu asymptomatischen echten Aneurysmen mit einer 5-Jahres-Über- lebensrate um $90 \%$. Die perioperative Mortalität wird inzwischen mit $<10 \%$ angegeben [4].

\section{Therapie und Verlauf}

Im vorliegenden Fall wurde der Patient zeitnah reoperiert. Intraoperativ fand sich ein sehr großes, echtes Aneurysma mit ausgedehnter Thrombosierung. Nach Aneurysmaresektion wurde der sehr große Defekt durch ein Rinderperikardpatch verschlossen und nochmals gerafft, um die LV-Größe und Mitralinsuffizienz zu reduzieren. Die TTE vor Verlegung ergab eine noch normale enddiastolische LV-Weite, aber eine weiter höhergradig reduzierte LV-EF um 35\% mit einem verbliebenen Restaneurysma von $18 \mathrm{~mm}$ Tiefe. Je nach weiterer Entwicklung der LVEF wird ggf. eine ICD-Implantation in Betracht gezogen werden müssen.

\section{Fazit für die Praxis}

Auch wenn durch die frühen Revaskularisationsstrategien mechanische Komplikationen nach Myokardinfarkten deutlich seltener geworden sind, sollte man diese nicht außer Acht lassen und routinemäßig die Patienten kurzfristig echokardiographisch nachuntersuchen, insbesondere wenn Risikofaktoren für eine Aneurysmabildung vorliegen. Herzinsuffizienz, embolische Schlaganfälle oder ventrikuläre Tachykardien nach Myokardinfarkt können durch Aneurysmabildung verursacht werden. Die TTE bleibt hier die erste Methode zur Diagnostik, hochauflösende Bildgebungen wie MRT und CT können dann zur Differenzialdiagnose zwischen Pseudoaneurysma und echtem Aneurysma beitragen, um die passende Therapiestrategie für den Patienten festzulegen.

\section{Korrespondenzadresse}

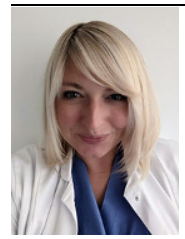

Dr. med. Vanessa RubeschKütemeyer

Klinik für Kardiologie,

Angiologie und internistische Intensivmedizin, Klinikum

Lippe Detmold

Röntgenstr., 32756 Detmold,

Deutschland

Vanessa.Rubesch-

Kuetemeyer@

klinikum-lippe.de

\section{Einhaltung ethischer Richtlinien}

Interessenkonflikt. V. Rubesch-Kütemeyer, A. Pula, M. Vlachojannis und S. Gielen geben an, dass kein Interessenkonflikt besteht. 
Für diesen Beitrag wurden von den Autoren keine Studien an Menschen oder Tieren durchgeführt. Für die aufgeführten Studien gelten die jeweils dort angegebenen ethischen Richtlinien. Für Bildmaterial oder anderweitige Angaben innerhalb des Manuskripts, über die Patienten zu identifizieren sind, liegt von ihnen und/oder ihren gesetzlichen Vertretern eine schriftliche Einwilligung vor.

\section{Literatur}

1. Bisoyi S, Dash AK, Nayak D, Sahoo S, Mohapatra R (2016) Left ventricular pseudoaneurysm versus aneurysm a diagnosis dilemma. Ann Card Anaesth 19:169-172

2. Frances C, Romero A, Grady D (1998) Left ventricular pseudoaneurysm. J Am Coll Cardiol 32:557-561

3. Hulten EA, Blankstein R (2012) Pseudoaneurysm of the heart. Circulation 125:1920-1925

4. Sattar Y, Alraies MC (2020) Ventricular aneurysm. https://www.ncbi.nlm.nih.gov/books/ NBK555955/?report=reader\#_article-31084_s4_. Zugegriffen:6. Sept. 2020

\section{Strahlentherapie bei Lungenkrebs: Wie lässt sich das Risiko für kardiale Ereignisse weiter minimieren?}

Eine aktuelle Studie definiert konkrete Schwellenwerte, ab wann für Patientinnen und Patienten mit nicht-kleinzelligem Lungenkrebs mit und ohne vorbestehende KHK ein erhöhtes Risiko für kardiale Folgeerkrankungen nach Strahlentherapie besteht.

Die Strahlentherapie ist eine etablierte Therapie, die auch in vielen Fällen bei nicht-kleinzelligen Lungenkarzinomen („,non small lung cancer"/NSCLC) zum Einsatz kommt. Wenn der linke Lungenflügel betroffen ist, erfolgt die Bestrahlung des linken und mittleren Brustkorbs (linksthorakal und mediastinal), was zu strahlenbedingten Veränderungen am Herzen führen kann. Zu nennen sind hier u.a. das Risiko einer koronaren Herzkrankheit (KHK), von strahlenbedingten Klappenerkrankungen oder auch einer Herzinsuffizienz. Doch das Risiko ist abhängig von der Strahlendosis und dem Bestrahlungsfeld. Eine aktuelle Studie zeigte, dass die Strahlendosis und das Strahlenvolumen am vorderen absteigenden Ast der linken Herzkranzarterie (Ramus interventricularis anterior) bei bisher herzgesunden Menschen mit NSCLC als Schwellenwert zur Risikostratifizierung herangezogen werden kann.

Bei der aktuellen in "JAMA Oncology" publizierten Arbeit handelt es sich um eine retrospektive Kohortenauswertung von 701 Patientinnen und Patienten mit lokal fortgeschrittenem NSCLC. Das mediane Alter der Studienteilnehmer betrug 65 Jahre, 50,8\% waren männlich. Nach Adjustierung gegen eine vorbestehende KHK und andere prognostische Faktoren zeigte sich eine Korrelation zwischen dem Dosisexpositionsvolumen am Ramus interventricularis anterior und schweren Koronarereignissen in Folge der Therapie. Bei Bestrahlung von $10 \%$ und mehr des vorderen absteigenden Asts der linken Herzkranzarterie mit 15 Gy oder mehr kam es zu signifikant mehr kardialen Ereignissen, auch Todesfällen, als unterhalb dieses Schwellenwerts. Diese Assoziation wurde bei Patientinnen und Patienten ohne kardiale Vorerkrankungen beobachtet, sie bestand jedoch nicht bei jenen mit vorbestehender KHK. Bei diesen vorerkrankten Patienten kam es hingegen bei Bestrahlung von $1 \%$ und mehr des linken Ventrikels mit 15 Gy zu einem erhöhten Risiko für kardiale Ereignisse.
"Das Wegweisende an dieser Studie ist, dass sie konkrete Schwellenwerte aufzeigt, ab wann für Patienten mit und ohne vorbestehende KHK ein erhöhtes Risiko für kardiale Folgeerkrankungen besteht. Diese Schwellenwerte müssen allerdings noch prospektiv-randomisiert überprüft werden", erklärt Univ.-Prof. Dr. Stephanie E. Combs, Pressesprecherin der Deutschen Gesellschaft für Radioonkologie (DEGRO). „Sie geben uns aber bereits jetzt eine erste Orientierung, wie wir durch eine optimierte Bestrahlungsplanung unter Berücksichtigung bestimmter kardialer Substrukturen das Risiko für strahlenbedingten Langzeitveränderungen am Herz reduzieren können.

Dennoch seien chronische Nebenwirkungen am Herzen nie ganz auszuschließen und können auch als Langzeitfolge auftreten, da immer mehr Lungenkrebspatientinnen und -patienten durch die Kombination von Strahlentherapie, Chemotherapie und Immuntherapie eine gute Überlebensprognose haben und oft in eine Art chronische Erkrankung überführt werden können, so dass sie viele Jahre mit der Krebserkrankung leben. „Das Management von kardialen Langzeitfolgen nimmt daher einen zunehmend wichtigen Stellenwert ein", so die Expertin.

\section{Literatur:}

Atkins K M, Chaunzwa $\mathrm{TL}$, Lamba $\mathrm{N}$ et al (2020) Association of Left Anterior Descending Coronary Artery Radiation Dose With Major Adverse Cardiac Events and Mortality in Patients With Non-Small Cell Lung Cancer. JAMA Oncol. doi:10.1001/jamaoncol.2020.6332

\section{Quelle: \\ Deutsche Gesellschaft für Radioonkologie e. V., www.degro.org}

